\title{
Dynamics of Nematogenic Molecules with Transverse Component of Dipole Moment
}

\author{
R. Douali ${ }^{a}$, G. Czechowski $^{b}$, Ch. Legrand ${ }^{a}$ AND J. JADŻYN ${ }^{b, *}$ \\ ${ }^{a}$ Université Littoral - Côte d'Opale, LEMCEL, BP 689, 62-228 Calais, France \\ ${ }^{b}$ Institute of Molecular Physics, Polish Academy of Sciences \\ M. Smoluchowskiego 17, 60-179 Poznań, Poland
}

(Received January 19, 2004)

\begin{abstract}
The paper presents the results of studies of the dielectric relaxation of nematic 4'-butyloxy-3-fluoro-4-isothiocyanatotolane, performed for different values of the angle between the probing electric field $\boldsymbol{E}$ and the macroscopic orientation of the sample (the director $n$ ) forced by the magnetic field $\boldsymbol{B}$. Due to a relatively large transverse component of the dipole moment of $4^{\prime}$-butyloxy-3-fluoro-4-isothiocyanatotolane, the studies allowed one to interpret consistently the dielectric relaxation spectra connected with the molecular rotation around the long axis.
\end{abstract}

PACS numbers: $64.70 . \mathrm{Md}, 77.84 . \mathrm{Nh}, 77.22 . \mathrm{Gm}$

\section{Introduction}

The dielectric relaxation is still a very useful method for studies of the molecular dynamics in isotropic liquid and liquid crystalline materials [1-6]. Assuming polar molecules to be a rigid body, interacting solely with an external electric field, the dynamics manifests itself as an electromagnetic energy absorption due to the changes of the molecules dipole moment projection on the field direction. In isotropic polar liquids, as the molecules rotate around their axes of symmetry corresponding to minimum and maximum of their momentum of inertia (the molecular long and short axes), two absorption bands can be observed in the dielectric relaxation spectrum.

*corresponding author; e-mail: jadzyn@ifmpan.poznan.pl 
A new axis of symmetry (the director $n$ ) appears when an external orienting field is applied to the nematic sample. Then, the long axis of mesogenic molecules forms with the director $n$ an angle $\Theta$, the mean value of which is a measure of the order parameter $S=\left\langle 3 \cos ^{2} \Theta-1\right\rangle / 2$. In such a case the mesogenic molecules rotate on the cone around the director $n$, and, as we discussed previously [7], a new absorption band appears at about $100 \mathrm{MHz}$ in the spectrum recorded for $\boldsymbol{E} \perp n\left[\varepsilon_{\perp}^{*}(\omega)\right]$.

The model of possible molecular rotations in oriented nematic liquid crystals proposed in [7] was based on the experimental results obtained for the mesogenic molecules in which the resultant dipole moment was roughly directed along the molecular long axis, i.e. the transversal component of the dipole moment was strongly reduced. Hence, the strength of the absorption band corresponding to the molecular rotation around the long axis was too low for its quantitative analysis. The data presented in this paper complete this matter: the experiment was performed for mesogenic molecules with an important transversal component of the dipole moment.

\section{Experimental}

The nematic liquid crystal 4'-butyloxy-3-fluoro-4-isothiocyanatotolane (BFTT)

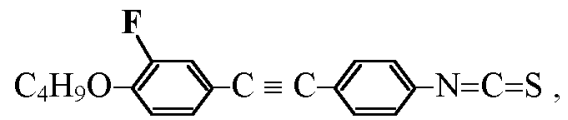

with the following sequence of phase transitions: $(\mathrm{Cr}) 62.6^{\circ} \mathrm{C}(\mathrm{N}) 71.8^{\circ} \mathrm{C}(\mathrm{I})$, was synthesized and purified at the Institute of Chemistry, Military Technical University, Warsaw. The nematic sample was placed in a plane cooper capacitor and oriented with the magnetic field $\boldsymbol{B}(1.2 \mathrm{~T})$. The sample thickness was $0.5 \mathrm{~mm}$. The dielectric relaxation spectra were recorded for different values of the angle $\alpha$ between the probing electric field $\boldsymbol{E}$ (which is perpendicular to the electrodes surface) and the director $n$ (i.e. the orienting magnetic field direction). The measurements were performed in the frequency range of $1 \mathrm{MHz}$ to $2 \mathrm{GHz}$ using an $\mathrm{HP} 4291 \mathrm{~B}$ Impedance Analyzer, at $65^{\circ} \mathrm{C}$.

\section{Results and discussion}

Figure 1 presents a quasi-continuous evolution of the dielectric relaxation spectrum of nematic BFTT from $\varepsilon_{\|}^{*}(\omega)$ to $\varepsilon_{\perp}^{*}(\omega)$, recorded at every $10^{\circ}$ angle step. As we observed previously for 6CHBT [7], the two spectra transform into each other softly. It is clearly seen in $2 \mathrm{D}$ picture that the dielectric anisotropy $(\Delta \varepsilon=$ $\varepsilon_{\|}-\varepsilon_{\perp}$ ) of BFTT changes its sign two times: at about $10 \mathrm{MHz}$ and $500 \mathrm{MHz}$. For low frequencies of the measuring electric field the dielectric anisotropy is positive, 

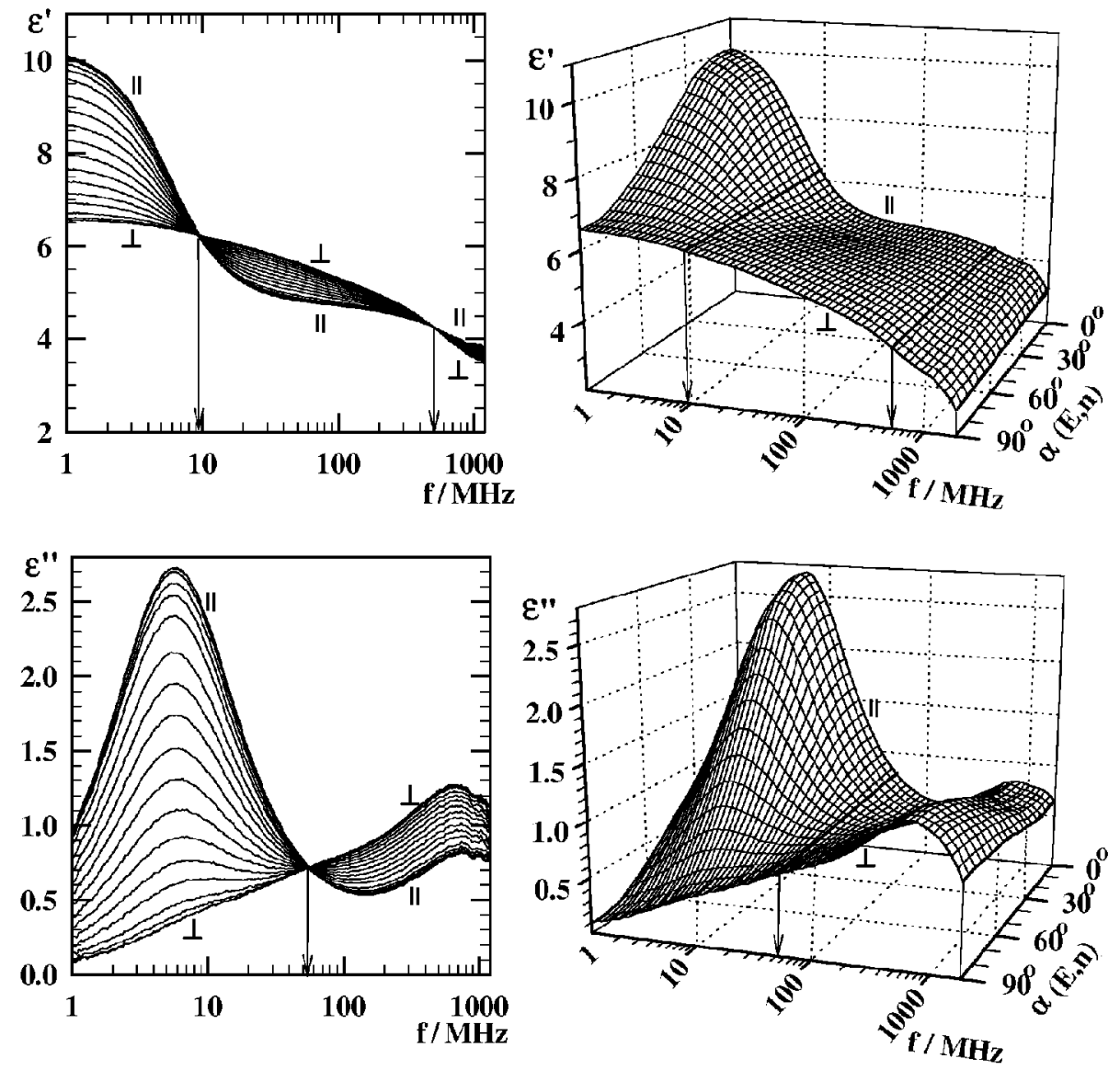

Fig. 1. The change of the dielectric relaxation spectra (2D and 3D) of nematic BFTT from $\varepsilon_{\|}^{*}(\omega)(\boldsymbol{E} \| n)$ to $\varepsilon_{\perp}^{*}(\omega)(\boldsymbol{E} \perp n)$ for the angle $\alpha(\boldsymbol{E}, n)$ step of $10^{\circ}$. The arrows point out the frequencies at which the dielectric characteristics show no angle dependence.

next between $10 \mathrm{MHz}$ and $500 \mathrm{MHz}$ is negative and for the higher frequencies once more positive.

As expected for the compound studied, due to the lateral substituted fluorine atom giving a transversal dipole moment of about $1 \mathrm{D}$, the strength of the dielectric absorption band situated at about $1 \mathrm{GHz}$ - corresponding to the molecular rotation around the long axis - is quite important and can be submitted to a quantitative analysis. In Fig. 2a the Cole-Cole plot transformation from $\boldsymbol{E} \| n$ to $\boldsymbol{E} \perp n$ is depicted for different values of the angle $\alpha$. In the plot corresponding to $\boldsymbol{E} \| n$ (Fig. 2b) one observes a good separated semi-circle corresponding to BFTT molecules rotation around the long axis. Figures $2 \mathrm{~b}$ and $\mathrm{c}$ present the resolution of the experimental $\varepsilon^{\prime \prime}$ vs. $\varepsilon^{\prime}$ dependences into elementary contributions, according to the model of possible molecular rotations in oriented nematic liquid crystal 

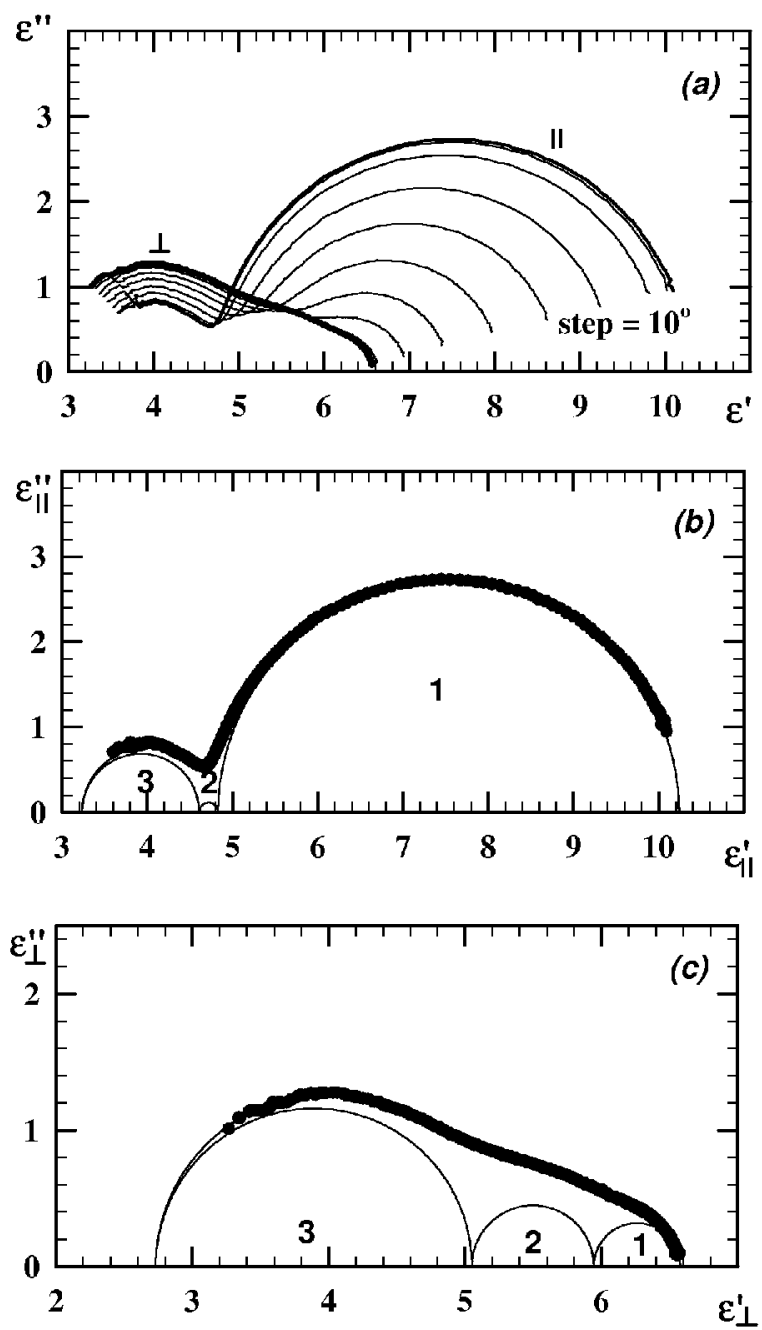

Fig. 2. Cole-Cole plots evolution from $\boldsymbol{E} \| n$ to $\boldsymbol{E} \perp \boldsymbol{n}$ (a) and the resolution of the extreme plots into elementary contributions ( $b$ and $c$ ).

presented in [7] and sketched in Fig. 3. The mesogenic molecules rotate around the three axes of symmetry: two of them concern the molecule itself - the short and long molecular axes (the band 1 and 3, respectively) and the third axis is the director $n$ (the band 2).

The resolution of the experimental dielectric relaxation spectra into elementary contributions was performed with the use of an empirical Cole-Cole formula [8]:

$$
\varepsilon_{a}^{*}(\omega)=\varepsilon_{a}^{\prime}(\omega)-\mathrm{i} \varepsilon_{a}^{\prime \prime}(\omega)=\varepsilon_{\infty}+\sum_{i=1}^{3} \frac{A_{i}}{1+\left(i \omega \tau_{i}\right)^{1-k_{i}}}, \quad a=\| \quad \text { or } \quad \perp
$$


where the $k_{i}$ parameter refers to the distribution of the relaxation times. For the limiting value $k_{i}=0$, the $i$-th reorientational process is described by a single relaxation time (the Debye-type process). $A_{i}$ and $\tau_{i}$ denote the dielectric strength and the relaxation time, respectively. $A_{i}, \tau_{i}$, and $k_{i}$ are the adjustable parameters in the procedure of the fitting of $\mathrm{Eq}$. (1) to the experimental data. The $\varepsilon_{\infty}$ is the high frequency value of the permittivity. From the fitting procedure results that, similarly to previously studied 6CHBT, the three absorption bands are very close to Debye-type $\left(k_{i} \approx 10^{-2}, i=1,2,3\right)$.
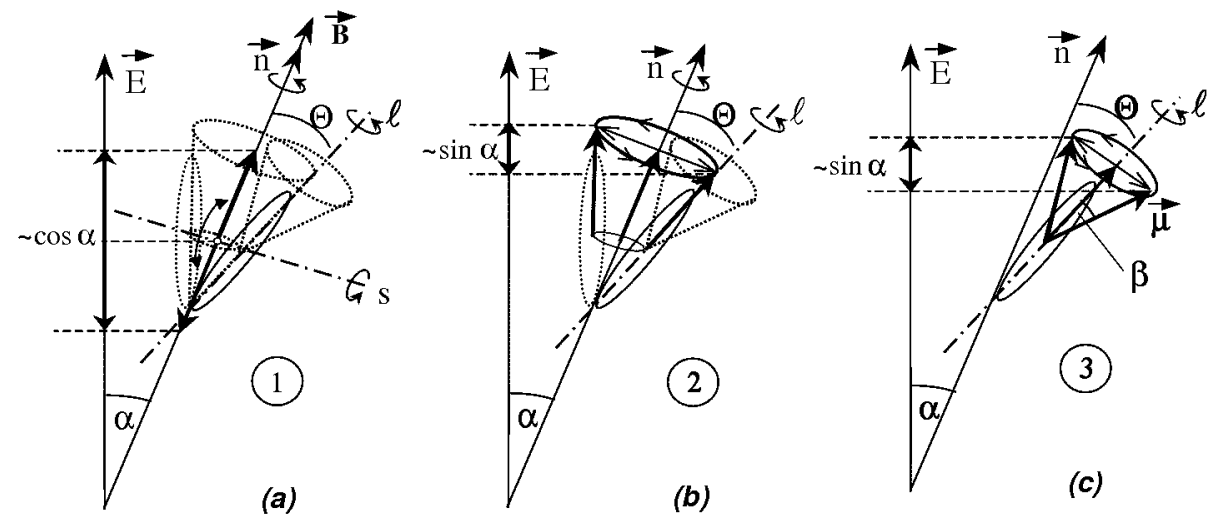

Fig. 3. Possible molecular rotations around the symmetry axes in oriented nematic liquid crystal, which cause a change of the dipole moment projection on the probing electric field $\boldsymbol{E}$ direction.

As results from the analysis of Fig. 3 , the dielectric relaxation spectra, both $\varepsilon_{\|}^{*}(\omega)$ and $\varepsilon_{\perp}^{*}(\omega)$, recorded for perfectly oriented nematic liquid crystal, should be composed of two elementary contributions. Namely, in the spectrum $\varepsilon_{\|}^{*}(\omega)(\boldsymbol{E} \| n)$ the energy absorption due to the rotations (a) and (c), and in the spectrum $\varepsilon_{\perp}^{*}(\omega),(\boldsymbol{E} \perp \boldsymbol{n})$ - rotations (b) and (c), can be observed. For the perfect orientation two conditions have to be fulfilled: (i) the axis corresponding to the minimum of the momentum of inertia (the long molecular axis) should be collinear to the axis of the maximum of magnetic susceptibility (when the magnetic field is used for molecular alignment), and (ii) there is no influence of the cell surfaces on the molecular orientation. Because, in general, the above conditions are not fulfilled, especially the second one, the most often the dielectric relaxation spectra are composed of three components, although, as seen in Fig. $2 \mathrm{~b}$, the band 2 in the $\varepsilon_{\|}^{*}(\omega)$ spectrum appears as a trace only. An appearance of the band 1 in the $\varepsilon_{\perp}^{*}(\omega)$ spectrum is certainly connected with the influence of the capacitor surfaces on the orientation of the mesogenic molecules, which, as a consequence, are not exactly parallel to the surfaces with their long axes.

Figures 4 and 5 present, respectively, the dependences of the strengths $A_{1}, A_{2}$, and $A_{3}$ and the relaxation times $\tau_{1}, \tau_{2}$, and $\tau_{3}$, corresponding to the elementary 


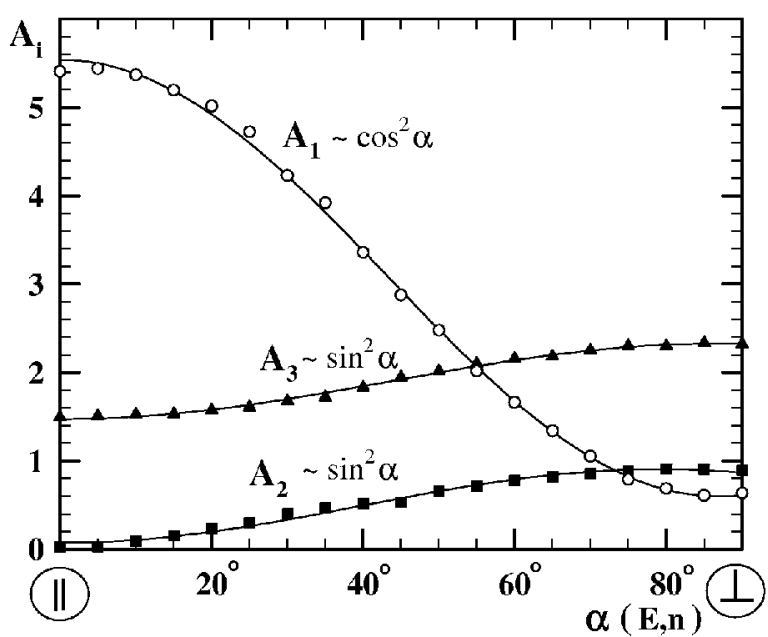

Fig. 4. The angle dependence of the strengths of the three elementary contributions to the dielectric relaxation spectra of nematic BFTT at $65^{\circ} \mathrm{C}$. The solid lines represent the functions $\cos ^{2} \alpha$ and $\sin ^{2} \alpha$, respectively.

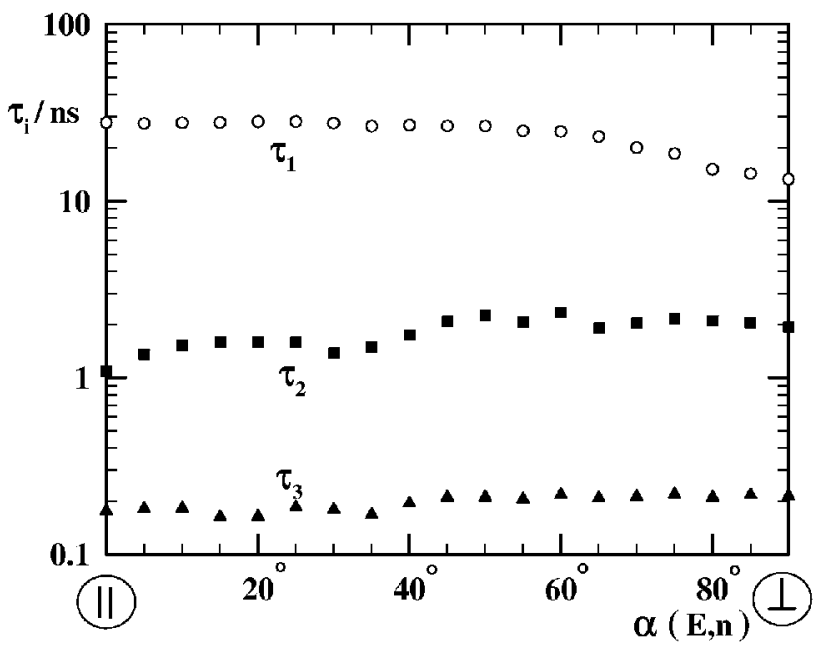

Fig. 5. The angle dependence of the relaxation times corresponding to the three elementary contributions to the dielectric relaxation spectra of nematic BFTT.

spectral contributions, on the value of the angle $\alpha(\boldsymbol{E}, \boldsymbol{n})$. Of course, the most important result concerns the angular behavior of $A_{3}$. The dielectric strength corresponding to the molecular rotation around the long axis changes as $\sin ^{2} \alpha$, which is in full agreement with our model (Fig. 3c). The angular dependences of $A_{1}$ and $A_{2}$ coincide with those previously observed for 6CHBT. Similarly to 6CHBT, the relaxation times $\tau_{1}, \tau_{2}$, and $\tau_{3}$ depend rather weakly on the angle $\alpha$. The relaxation 
time $\tau_{1}$, corresponding to the molecular rotation around the short axis (strictly, it is the rotation around the axis perpendicular to the director $\boldsymbol{n}$ ) points out for a tendency for acceleration of this rotation for $\alpha$ approaching $90^{\circ}$. The effect is probably due to the essential reduction of the interaction between the molecular dipole moment and the probing electric field in the configuration close to $\boldsymbol{E} \perp \boldsymbol{n}$.

\section{Acknowledgment}

The work was supported by the Centre of Excellence for Magnetic and Molecular Materials for Future Electronics within the European Commission Contract No. G5Ma-CT-2002-04049.

\section{References}

[1] B.K.S. Scaife, Principles of Dielectrics, Clarendon Press, Oxford 1998.

[2] S. Urban, A. Würflinger, in: Advances in Chemical Physics, Eds. I. Prigogine, S.A. Rice, Vol. 98, Wiley, New York 1997, p. 143.

[3] A. Kozak, J.K. Mościcki, Liq. Cryst. 12, 377 (1998).

[4] H. Kresse, in: Advances in Liquid Crystals, Ed. G.H. Brown, Vol. 6, Academic Press, New York 1983, p. 109.

[5] G. Williams, in: The Molecular Dynamics of Liquid Crystals, Eds. G.R. Luckhurst, S.A. Veracini, Kluwer Academic Press, Dordrecht 1994, p. 438.

[6] A. Oka, G. Sinha, C. Glorieux, J. Thoen, Liq. Cryst. 31, 31 (2004).

[7] J. Jadżyn, G. Czechowski, R. Douali, Ch. Legrand, Liq. Cryst. 26, 1591 (1999).

[8] K.S. Cole, R.H. Cole, J. Chem. Phys. 9, 341 (1949). 